\title{
Reflexiones
}

\section{Aportes a un debate sobre el futuro de la integración latinoamericana y del Mercosur}

\author{
Félix Peña ${ }^{1}$
}

* Cómo citar este artículo: Peña, F. (2021). Aportes a un debate sobre el futuro de la integración latinoamericana y del Mercosur. Relaciones Internacionales, 30(60), 122. https://doi.org/10.24215/23142766e122

\section{Comentarios introductorios}

El actual es un mundo más poblado y más conectado. Por las tendencias que se observan, estos son rasgos que tenderán a acentuarse en las próximas décadas. En un mundo con estas características, la Argentina puede -si se lo propone- desarrollar relaciones comerciales normales y eventualmente intensas con todos los países, especialmente aquellos con los cuales se comparten intereses concretos. Pero se trata de una estrategia que implicaría consolidar la relación estrecha preferencial que el país pactó en 1991 con sus socios del Mercosur, a partir del acuerdo que pocos años antes habían celebrado entre la Argentina y el Brasil los Presidentes Raúl Alfonsín y José Sarney.

Pero una estrategia de inserción activa en todos los ámbitos del sistema comercial internacional como la que podrían desarrollar, si se lo proponen, tanto la Argentina como el Mercosur y los países latinoamericanos que compartieran tal enfoque, requiere tener un diagnóstico permanentemente actualizado de los factores que permiten anticipar cambios significativos, tanto en el plano global como en los múltiples y diversos planos regionales. Requiere, en especial, de una eficaz organización en el plano gubernamental y en el de la

\footnotetext{
${ }^{1}$ Director de la Fundación ICBC y de la Maestría en Relaciones Comerciales Internacionales de la UNTREF. Ver todas sus publicaciones en www.felixpena.com.ar.
}

Editor: Juan Alberto Rial, Instituto de Relaciones Internacionales Facultad de Ciencias Jurídicas y Sociales (Universidad Nacional de La Plata)
Entidad editora: Relaciones

Internacionales, es una publicación del Instituto de Relaciones Internacionales (Facultad de Ciencias Jurídicas y Sociales (Universidad Nacional de La Plata Argentina)
s.unlp.edu.ar/RRII-IRI Vol 30 - no 60/2021 
sociedad en su conjunto, así como también de una activa política de cooperación con otras naciones y, por cierto, no solo las de la región latinoamericana.

Entre otros, cambios tecnológicos (en todos los planos), climáticos y culturales (valores, preferencias, costumbres) incidirán en los desplazamientos de ventajas competitivas y, por ende, en el desarrollo del comercio de bienes y de servicios entre los países y regiones. Asimismo, el actual es un mundo con marcadas diversidades de consumidores empoderados, como resultado de una creciente población en muchos países, y de una clase media urbana bien informada sobre sus múltiples opciones para obtener los recursos, bienes y servicios que eventualmente necesite o prefiera. Un mundo con marcadas diversidades culturales en múltiples planos requerirá potenciar las que caracterizan a la Argentina y a América Latina. Será también un mundo más contagioso, ya sea en el plano de las ideas, de los valores, de las políticas e incluso de las pestes (como lo demuestra la experiencia de la denominada pandemia del coronavirus).

\section{La importancia de las usinas de pensamiento orientadas a la acción}

Las denominadas usinas o espacios de pensamiento orientados a la acción tienen importancia en el desarrollo de las relaciones comerciales internacionales de cualquier país. Reflejan el vínculo para que un país desarrolle desde su propia perspectiva, entre la reflexión nutrida en la experiencia y en la acción concreta con el fin de lograr efectividad y eficacia en su inserción internacional y en las de sus empresas. Se basan en la capacidad para entender las realidades sobre la base de la experiencia. Su valor práctico aumenta por la creciente complejidad y dinámica que tienen hoy las relaciones comerciales entre naciones.

No hay modelos únicos sobre cómo encarar el desarrollo de tales usinas en un país determinado, pero se observan rasgos comunes en instituciones existentes. Dos son los más destacables: el primero es la inclusión, en sus agendas de debates y análisis, de cuestiones relevantes en las estrategias de inserción internacional; y el segundo es la conexión de sus agendas con las que desarrollan usinas similares en otros países, encarando incluso agendas comunes. La relevancia de las cuestiones que se privilegien, así como la densidad de las redes que se enhebren y la conectividad de las agendas, pueden hacer de tales redes un factor fundamental para la construcción de una interdependencia cooperativa entre países, por ejemplo, de una misma región, especialmente en función de sus respectivas inserciones en el sistema internacional global.

También es posible observar elementos comunes en las metodologías de trabajo que emplean distintas usinas conectadas entre sí. Un elemento común es el encarar sus agendas con la participación de múltiples protagonistas que expresan diversidades en el plano de roles sociales, en el generacional y en el de visiones sobre las realidades. Otro elemento es el concentrar el análisis en pocas cuestiones relevantes para la agenda internacional del momento y que, por su complejidad, requieran ser apreciadas en múltiples perspectivas disciplinarias. Y un tercer elemento común es el de aportar ideas concretas y útiles en el plano de acciones que aborden cuestiones relevantes de la agenda internacional de un país o de un grupo de países. 
Son conversatorios, muchas veces virtuales, entre protagonistas con distintos roles sociales en la reflexión y en la acción, y con distintas visiones sobre cómo operar en las realidades. También tiene importancia la forma de encarar la conversación entre los participantes. Las mejores reuniones son aquellas en las que la agenda contenga pocos módulos temáticos, un moderador que sea un verdadero provocador del debate, presentaciones iniciales cortas (lo ideal son unos diez minutos; obviamente, sin lectura de textos escritos; y, en lo posible, con pocos cuadros estadísticos) y luego un buen período de conversación con intervenciones cortas (lo ideal son unos tres minutos como máximo) y con un protagonismo activo del moderador-provocador. Normalmente son reuniones que no requieren conclusiones formales. Las conclusiones las extrae cada participante en función de su respectiva agenda como especialista, analista o protagonista en las relaciones internacionales de su respectivo país.

Estas usinas pueden ser más eficaces en la medida que reúnan dos requisitos. Uno es que quien esté en el plano de la acción gubernamental o empresarial, demuestre interés en recibir orientaciones de quienes están en el plano de la reflexión y de la experiencia. El otro es que quien esté en el plano del pensamiento esté dispuesto a colocarse en el lugar de quien está en el plano de la acción -o sea en "el campo de batalla-y de plantearle sugerencias fundadas en experiencias que respondan a la hipotética pregunta “¿cómo lo hago?”.

\section{La construcción del espacio regional latinoamericano}

La construcción de espacios regionales multinacionales, como es el caso de América Latina, con objetivos políticos de largo plazo que trascienden al plano económico y con reglas de juego favorables al desarrollo del comercio, del empleo y de la inversión productiva, no es tarea fácil. Lo demuestra precisamente la experiencia latinoamericana, pero también, entre otras, la europea; en especial cuando tal construcción se refleja en procesos que son menos sencillos de desarrollar que lo imaginado en sus momentos fundacionales.

Quizás sea por ello por lo que hoy los países parecen estar menos inclinados a la idea de una integración económica con naciones del propio espacio geográfico, si es que ella supone disciplinas rígidas, superación de su propia identidad nacional y, por sobre todo, cesión definitiva de soberanía.

Se evidencia en las crisis que atraviesan algunos de los principales procesos de integración encarados en las últimas décadas. Ejemplos más notorios se encuentran en el Mercosur y en la Unión Europea (UE). No son, por cierto, procesos similares ni en sus rasgos principales ni en su profundidad. Tampoco lo son sus respectivas crisis. Pero ellas evidencian a veces una creciente distancia entre los avances logrados y las expectativas generadas en cuanto a sus posibles resultados.

En ambos casos se observan factores que generan incertidumbres sobre su desarrollo futuro. Ello es evidente en el caso de la UE, como consecuencia de los efectos aún imprecisos del Brexit y de su potencial pleno impacto en el proceso regional de integración. En el caso del Mercosur, si bien el respectivo país miembro ha ratificado su voluntad de continuar con 
su desarrollo, siguen en pié muchos interrogantes sobre cómo se abordarán cuestiones que inciden en su eficacia y en su legitimidad social.

Es razonable imaginar que, hacia adelante los acuerdos de integración, tanto los regionales como los birregionales en sus distintas modalidades -tal es el caso del previsto acuerdo entre el Mercosur y la UE-, seguirán impactados por las incertidumbres que predominen en el contexto internacional.

Tales incertidumbres derivan, entre otros factores, de los efectos de las nuevas configuraciones del poder mundial; de la mayor conectividad entre países, empresas y consumidores con múltiples opciones; y de la proliferación de fracturas étnicas, ideológicas y emocionales. Ellas acrecientan la importancia para todos los protagonistas de contar con estrategias de inserción internacional que estén actualizadas, que sean relativamente confiables y que abarquen el mayor número de países.

Se está entrando en una fase de relaciones internacionales policéntricas, en la que se pueden tornar obsoletos paradigmas, conceptos y enfoques teóricos del pasado. En tal fase será necesario tener una visión actualizada del propio concepto de integración económica entre diversos países. Implica tener claro por qué un país se asocia en forma permanente con otro u otros países (dimensión existencial de la integración) y cómo hacerlo (dimensión metodológica).

Imaginar la integración en términos similares a los que han predominado en otros momentos históricos, especialmente en el plano económico, no parecería ser algo conveniente. En tal perspectiva, una visión dogmática de lo que debe ser el grado de perfección que tenga una zona de libre comercio o una unión aduanera, suena a algo antiguo.

La que está emergiendo ahora parecería ser una fase de alianzas múltiples, simultáneas y flexibles, con una gran dinámica en su desarrollo. Una fase en la que un país, cualquiera que sea su dimensión, pueda tener dificultades para navegar con eficacia y para lograr sus visiones y objetivos, si es que no tiene un diagnóstico correcto, sobre su ubicación y sobre sus posibilidades efectivas en la competencia por el poder y por los mercados internacionales.

\section{Las agendas de inserción comercial externa de países latinoamericanos}

Varias cuestiones novedosas se pueden destacar en la agenda de prioridades para la inserción comercial internacional de países latinoamericanos. Tienen que ver con la necesidad de actualizar y renovar estrategias de proyección al mundo de bienes y de servicios que un país está en condiciones de producir con calidad y eficacia, sobre la base de ventajas competitivas y, en especial, de dotación de recursos naturales, talentos y creatividad, y de diversidad de su desarrollo industrial y tecnológico.

Algunas de las principales cuestiones que se deberían observar en las agendas de inserción comercial internacional de países latinoamericanos tendrían que ser las siguientes:

- $\quad$ en el plano global, entender e incidir en la necesaria renovación del sistema multilateral del comercio mundial, institucionalizado en la OMC y, a la vez, lograr la vinculación 
de cada país con la creciente red de acuerdos comerciales preferenciales de alcance regional e inter-regional;

- en el plano regional latinoamericano, tener una participación activa en la adaptación del Mercosur a nuevas realidades del comercio internacional, incluidos tanto la necesaria articulación entre sus países miembros y los de la Alianza del Pacífico, como el pleno aprovechamiento de todo el potencial del Tratado de Montevideo de 1980 que creó la ALADI, y

- en el plano nacional, lograr un efectivo desarrollo de las estrategias de inserción competitiva en el comercio mundial de bienes y de servicios, especialmente de las pymes y de las empresas de nicho.

Las reformas a la OMC son hoy prioritarias. Es un marco institucional clave para preservar reglas básicas para el comercio internacional, tales como la del principio de no discriminación en las políticas arancelarias y en otras prácticas comerciales.

Parecería existir consenso entre los países miembros de la OMC sobre la necesidad de adaptar sus reglas a las nuevas realidades del comercio internacional. Muchas de ellas provienen de cuando se negoció en 1947 el Acuerdo General sobre Aranceles y Comercio (GATT). Ese era un momento de las relaciones internacionales sustancialmente distinto al actual. Pocos países participaron de las negociaciones y uno tenía por su poder relativo un protagonismo decisivo. El interés dominante era lograr un sistema de comercio global orientado por reglas y centrado en pocos principios. El de la no discriminación era uno fundamental. Y una regla principal que debía garantizarlo era la del artículo XXIV del GATT, que en la práctica permitía excepciones a tal principio, a través de dos modalidades: la de la unión aduanera y la zona de libre comercio.

Hoy la OMC -sucesora del GATT y que incorporó sus principales reglas, incluida la del citado artículo XXIV- tiene 164 países miembros. El poder relativo entre los distintos países es hoy diferente al del momento fundacional y ninguno individualmente tendría, aunque así lo crea, suficiente capacidad como para aspirar a imponer las reglas del comercio internacional sólo en función de sus intereses nacionales.

Por lo demás, la dinámica del comercio mundial en un mundo con más protagonistas y una creciente conectividad entre los espacios económicos nacionales y regionales, tornan más necesaria que nunca la existencia de reglas de juego cuyo cumplimiento no dependa sólo de la voluntad de cada país o de un grupo de países.

Pero el predominio del consenso como principal criterio para cambiar las reglas existentes o para aprobar nuevas reglas torna muy difícil el desarrollo exitoso de todo intento de adaptar el sistema de la OMC a nuevas realidades. La experiencia de la Rueda Doha así lo demostraría.

Una contribución valiosa de la Cumbre del G20 en Buenos Aires (2018) fue la de constatar que el sistema multilateral del comercio no está cumpliendo sus objetivos y que existen posibilidades de mejorarlo. Por ello se reconoce la necesidad de hacer las reformas necesarias para mejorar su funcionamiento. 
Un factor adicional incide en la importancia práctica que hoy tiene la cuestión de las reformas orientadas a modernizar la OMC. Un mecanismo de solución de controversias de hecho paralizado afecta significativamente la eficacia de las reglas multilaterales del comercio mundial. El sistema puede quedar expuesto, entonces, al criterio discrecional de los países miembros, sobre todo de los que tienen un mayor poder relativo.

Pero para abordar las reformas de la OMC, un problema significativo resulta del hecho de que no todos los países miembros del sistema comparten similares criterios para definir cuáles deban ser cuestiones prioritarias a abordar. A la hora de construir los necesarios consensos no es este un problema menor.

A su vez, en el plano regional latinoamericano, la reforma y actualización del Mercosur, así como su articulación con la Alianza del Pacífico, también tiene una fuerte prioridad. Todo indica que, por razones políticas, lo conveniente será alcanzar tal objetivo sin que sea necesario reformar el Tratado de Asunción, lo que podría plantear dificultades internas en algunos de los países miembros. Esto es factible si se logra el predominio de enfoques prácticos y no dogmáticos, especialmente sobre lo que debe ser una unión aduanera o una zona de libre comercio. La combinación entre sentido político, pragmatismo económico y flexibilidad jurídica permitiría lograr resultados concretos, asegurando a la vez la necesaria previsibilidad de las reglas que se pacten.

Y en el plano regional latinoamericano, el Tratado de Montevideo de 1980 brinda con su figura de los "acuerdos de alcance parcial", los instrumentos necesarios para el desarrollo de una estrategia inteligente de articulaciones productivas de alcance sectorial.

\section{La ALADI y su futuro en la construcción de un regionalismo latinoameri- cano insertado en el mundo}

La Asociación Latinoamericana de Integración (ALADI) fue creada en 1980 en sustitución de la Asociación Latinoamericana de Libre Comercio (ALALC). Ésta a su vez había sido creada en 1960, a fin de desarrollar una zona de libre comercio que debía perfeccionarse en un plazo de 12 años. Ni su formato -zona de libre comercio- ni los plazos estipulados pudieron ser cumplidos. En realidad, cuando se comenzó la negociación del Tratado de Montevideo de 1960, el objetivo original de los países era impulsar acuerdos preferenciales comerciales, especialmente de alcance sectorial, a fin de sustituir los acuerdos bilaterales desarrollados en la década de 1930.

La ALADI tiene un potencial adaptado a los actuales requerimientos de la cooperación e integración latinoamericana, concebida en una perspectiva estratégica de "convergencia en la diversidad".

Su pleno aprovechamiento no depende necesariamente de una acción colectiva de todos sus países miembros. El instrumento de los acuerdos de alcance parcial previsto en el Tratado y reglamentado por la resolución 2 de la Conferencia en 1980 es el que brinda en la actualidad un mayor potencial, en forma compatible con la normativa del sistema multilateral del comercio internacional (GATT-OMC). 
Desde que fue lanzada en 1980 -en sustitución de la Asociación Latinoamericana de Libre Comercio (ALALC), creada para desarrollar una zona de libre comercio a perfeccionarse en un plazo de 12 años-, la ALADI tiene por lo menos dos funciones a fin de desarrollar "un área de preferencias económicas", que es su principal objetivo como contribución a la idea más amplia de integración económica regional.

Una primera función es la de brindar un marco legal para la celebración de acuerdos entre todos (los acuerdos de alcance regional - artículo 6) o al menos entre dos o más de sus países miembros, pero no todos (los acuerdos de alcance parcial - artículos 7 a 14). En tal caso sólo se requiere la aprobación de los países participantes en el respectivo acuerdo. Dichos acuerdos (regionales o parciales) deben contribuir al desarrollo de un mercado común (objetivo sin plazo definido y a lograrse "en forma gradual y progresiva") entre sus países miembros, precisamente por el desarrollo de preferencias económicas.

La otra función es la de brindar un marco institucional para avanzar en el cumplimiento de sus objetivos, a través de los órganos representativos de los Estados y del papel que cumple su Secretaría General, que funciona en Montevideo.

La primera función permite brindar sustento legal, dentro y entre los países, y también en el sistema multilateral del comercio mundial (antes el GATT y a partir de 1995, la OMC, sea por el artículo XXIV de las reglas del GATT-OMC o por la denominada Cláusula de Habilitación, mucho más flexible), a las preferencias comerciales que se otorgan los países miembros entre sí. En los acuerdos de alcance parcial, tales preferencias se extienden sólo a los países participantes, pero eventualmente otros o todos los países miembros de la ALADI pueden requerir participar. Una regla general establecida por el Tratado es la de la cláusula de la nación más favorecida (artículo 44), que obliga a extender a todos los miembros cualquier ventaja arancelaria que no se hubiere otorgado en el marco de un acuerdo de alcance parcial.

Los países miembros tienen una representación permanente en la ALADI. Otra función que puede cumplir la ALADI es la de que tal representación sirva para construir una red de acuerdos comerciales y de integración con otros países de la región, complementando las acciones que se desarrollen en el plano bilateral con el respectivo país. En tal caso, bien concebida, la Secretaría General podría cumplir una función de impulso y de apoyo técnico a las gestiones que se realicen.

En síntesis, la ALADI puede servir como marco institucional que permite, por un lado, dar cobertura jurídica a acuerdos con elementos de preferencias comerciales y económicas que se desarrollen con todos o algunos países miembros y eventualmente impulsarlos; y, por el otro lado, brindar un espacio de interacción con otros países con objetivos de promover y facilitar, entre otros objetivos, el desarrollo del comercio y de la complementación económica; sin perjuicio de que a través del tiempo puedan tener un alcance que cubra objetivos más generales y ambiciosos (por ejemplo, un mercado común) y que se extienda a todos los países miembros.

Para las empresas interesadas en construir o en participar en redes transnacionales entre empresas de la región cubierta por la ALADI, este marco institucional puede servir de 
fuente de información sobre las acciones preferenciales que se realicen en la región y, también, como ámbito para impulsar acuerdos gubernamentales (de alcance regional o parcial) que sean funcionales a sus objetivos en otros países de la región.

Especialmente, bien interpretado, el instrumento de los acuerdos de alcance parcial puede ser funcional al tejido de redes sectoriales de complementación e integración productiva. Para las empresas, especialmente pymes, los acuerdos de alcance parcial, con modalidad de complementación económica, pueden ser instrumentos funcionales para una estrategia sectorial de complementación con empresas de otros países de la ALADI.

Bien concebidos, los acuerdos de alcance parcial -especialmente de complementación económica y con alcance sectorial- permiten lograr equilibrios razonables entre dos demandas a veces contradictorias, tanto de las empresas como de los respectivos gobiernos. Una es la demanda de flexibilidad en las reglas de juego que se apliquen para desarrollar las estrategias sectoriales de complementación productiva. La otra es la demanda de previsibilidad sobre la aplicación de las respectivas reglas de juego -especialmente en lo referente a la estabilidad en las condiciones de acceso a los respectivos mercados- pactadas en el acuerdo de alcance parcial. Esta última es la que más puede ser requerida por las empresas llamadas a invertir en función del mercado ampliado por el respectivo acuerdo de alcance parcial.

Los acuerdos de alcance parcial son negociados por los países interesados, con conocimiento de los demás países miembros. No requieren ser aprobados por los órganos de decisión de la ALADI. La Secretaría General tiene además una función de registro y seguimiento de los acuerdos que se concluyan. En la página web de la ALADI están todos los textos de los acuerdos concluidos.

La ALADI es, en tal sentido, un marco institucional apropiado para avanzar en el desarrollo de acuerdos de complementación económica, con alcance sectorial o multisectorial, por ejemplo, entre países del Mercosur y de la Alianza del Pacífico y otros países latinoamericanos, sean o no miembros de la ALADI.

\section{Prioridades para adaptar el Mercosur a nuevas realidades}

Lo esencial de lo que se pactó en Asunción el 26 de marzo de 1991, al firmarse el Tratado por el que se creó el Mercosur, está reflejado en la reciprocidad de las preferencias comerciales que se otorgaron entre sí los países miembros y, en particular, los de las dos principales economías del espacio de integración regional que se creaba.

"Te doy y me das" sería una forma popular de expresar tal elemento esencial del Mercosur. Explícitamente se optó por una unión aduanera y no por una zona de libre comercio. Los artículos 1 y 5 del Tratado contienen los elementos esenciales del pacto entre los países socios. Sin embargo y sobre todo, la idea central del alcance de tal pacto se refleja en el artículo 2, que establece la reciprocidad como compromiso principal ("El Mercado Común estará fundado en la reciprocidad de derechos y obligaciones entre los Estados Parte"). 
Está claro que el Tratado de Asunción implicó mucho más que un sistema de preferencias comerciales. Sus dimensiones políticas y económicas básicas eran tan o más profundas que las preferencias comerciales. Penetraban hondo en la historia de las relaciones entre un grupo de países latinoamericanos, a veces más marcadas por tendencias al conflicto que a la cooperación. El Tratado implicó, además, afirmar la idea de trabajar juntos para promover una inserción internacional inteligente, efectiva y eficaz de cada uno de los países miembros del Mercosur.

Treinta años después de la firma del Tratado de Asunción, siguen teniendo vigencia los elementos que hacen a la dimensión existencial del Mercosur; esto es, el por qué trabajar juntos. No se cuestiona la necesidad de que este grupo de naciones contiguas que comparten su pertenencia a una región de fuerte potencial y ricas diversidades, trabajen juntas para potenciar su desarrollo económico y social, fortalecer sus sistemas políticos, y logar una inserción en el sistema internacional funcional a sus intereses y posibilidades.

Las diferencias se observan en la dimensión metodológica; esto es, el cómo trabajar juntos. Son diferencias naturales en todo proceso voluntario de integración entre naciones soberanas que no se plantean el dejar de serlo. El objetivo, por el contrario, es compartir el ejercicio de sus respectivas soberanías sin perder sus individualidades como naciones.

Las instituciones y reglas que se crean implican disciplinas colectivas que permitan llevar adelante un proceso de construcción de la integración procurada. La experiencia internacional indica que tal proceso de construcción puede llevar tiempo, incluso más que el imaginado. De ahí es que pueda requerirse adaptar los pasos de aproximación a los objetivos procurados. Incluso el camino hacia las metas perseguidas puede necesitar de adaptaciones frecuentes. La experiencia europea ha sido muy elocuente en tal sentido.

Los problemas no provienen, entonces, de la necesidad de una adaptación continua de un proceso de integración voluntario entre naciones a cambios frecuentes de las realidades, tanto en los países participantes como en el entorno regional y global en el que se insertan. Los verdaderos problemas pueden provenir, en cambio, de las deficiencias de los métodos que se emplean para eventualmente reorientar el recorrido del camino trazado o para revisarlo cuando fuere necesario por el peso de las realidades. Son problemas, incluso, que pueden exteriorizar deficiencias en los métodos empleados para concertar decisiones conjuntas o para asegurar su puesta en práctica. O pueden estar exteriorizando deficiencias en la elaboración de las respectivas posiciones nacionales con respecto a los desafíos que surgen de la evolución de las realidades. Y también pueden devenir, entre otros factores, de deficiencias de diagnósticos sobre tales realidades, que pueden provenir, ya sea de los actores gubernamentales como del propio sector empresario, o de los múltiples y diversos sectores sociales.

Las diferencias metodológicas tienen efectos más complejos si se traducen en diferencias existenciales. En cierta forma esta es una de las lecciones del Brexit, al menos desde la perspectiva de quienes lo impulsaron. Así puede ocurrir, por ejemplo, cuando se considera que hay fallas en el diagnóstico de qué es lo que anda mal en un proceso de integración. En 
tal caso, si un país percibe dificultades para introducir modificaciones en la dimensión metodológica y considera que ello puede afectar sus intereses nacionales, tiene siempre la opción existencial de retirarse del respectivo proceso de integración.

Las deficiencias metodológicas pueden ser resueltas con modificaciones a las reglas y disciplinas comunes pactadas, incluidas -si fuere necesario- las del propio pacto constitutivo.

En la perspectiva de lo antes esbozado, cobra toda la importancia que tiene en un proceso de integración como el del Mercosur el buen diagnóstico que se pueda realizar sobre cuáles son sus dificultades prácticas para navegar un mundo en continuo proceso de cambio. Es un diagnóstico que requiere tener en cuenta tanto la perspectiva nacional de cada uno de los países que participan del proceso, como la propia perspectiva común desde el proceso de integración, en este caso, desde el propio Mercosur. Y son diagnósticos en los que se ponen en evidencia la intensidad y la calidad de la interacción entre los múltiples actores participantes, lo que incluye, en especial, el aporte de los grupos de pensamiento y reflexión orientados a la acción.

Pero asumiendo que los diagnósticos sean correctos, no sería por cierto suficiente. Lo que realmente se requiere para encarar las crisis metodológicas en un proceso de integración, sobre todo si tienen potencial para derivar en crisis existenciales, son mecanismos eficaces de concertación de intereses nacionales en función de los intereses percibidos como comunes. Y ello implica, por sobre todos los otros factores, el liderazgo político en el más alto nivel de los países involucrados, la capacidad de concertación del órgano común del proceso de integración, y un protagonismo activo de los respectivos múltiples sectores económicos y sociales.

Los momentos críticos de los procesos de integración, como son los casos de la Unión Europea y del Mercosur, entre otros, ponen de manifiesto que es en la capacidad de concertación del órgano común y del liderazgo político en los países miembros en donde residen los factores más relevantes para una metodología eficaz de integración que permita lograr los objetivos procurados y evitar la recurrencia o el éxito de eventuales crisis existenciales.

A comienzos del año 2021, parece oportuno destacar tres cuestiones relevantes para modernizar el Mercosur y restablecer un grado aceptable de su credibilidad y de su eficacia. La primera cuestión se refiere a las metodologías de apertura de los respectivos mercados con su incidencia en las negociaciones comerciales internacionales. La segunda se refiere a la metodología institucional -incluida la capacidad para ejercer la necesaria función de concertación de los intereses nacionales- que se aplica para la adopción de decisiones conjuntas, que también inciden en el desarrollo de la agenda de negociaciones comerciales con otros países. Y la tercera se refiere la metodología que se emplea para asegurar que el proceso de integración esté basado en reglas de juego comunes y, por ende, orientado a ellas.

Por supuesto, hay otras cuestiones relevantes a abordar. Pero las tres mencionadas son las que, tras casi treinta años de desarrollo del Mercosur, parecería conveniente tener presente, especialmente en las conversaciones al más alto nivel político. 